\title{
Looking for Dark Matter with Gravitational Waves
}

\author{
Physicists are building a prototype tabletop detector to capture \\ gravitational waves at higher frequencies than LIGO can and to possibly \\ spot waves from annihilating axions.
}

\author{
By Erika K. Carlson
}

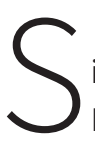
earches for new particles often evoke images of high-energy accelerators that stretch for miles.

But small-scale experiments that use high-precision measurement techniques can also expand our knowledge of fundamental particles. In early June, researchers reported a slew of experimental hunts for exotic new physics in a session of the virtual meeting of the APS Division of Atomic, Molecular Optical Physics (DAMOP). Among them was Andrew Geraci of Northwestern University in Illinois, who described a prototype tabletop gravitational-wave detector that he and colleagues have started building. The device would use a levitated
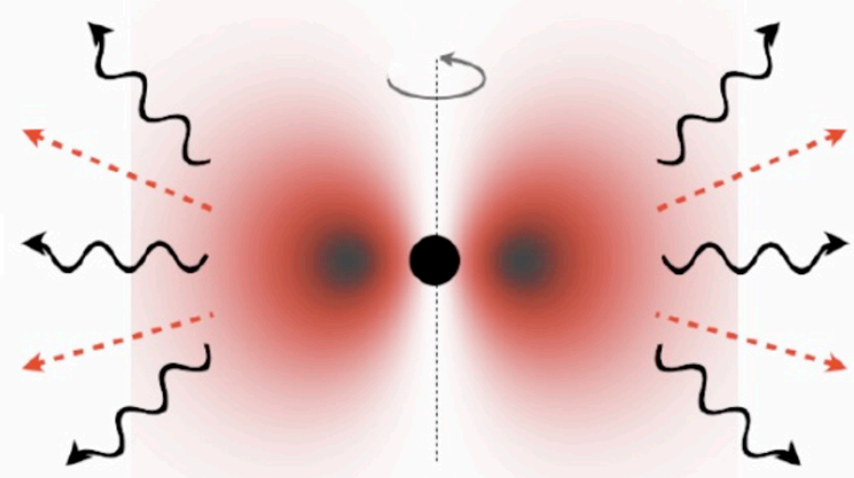

Figure 1: If axions exist, huge numbers of them would likely form clouds around black holes. Annihilating axions in these clouds could produce high-frequency gravitational waves detectable from Earth.

Credit: Asimina Arvanitaki/Stanford University nanoparticle to detect gravitational waves at frequencies beyond the range of sensitivity of giant laser interferometers like LIGO. The expanded frequency range might eventually let the researchers spot axions, a candidate dark matter particle, as well as other yet-unknown sources of gravitational waves in the cosmos.

"Every time we have looked at the Universe using a new frequency of the electromagnetic spectrum, we learned something new," says Nancy Aggarwal, a postdoc at Northwestern and a member of the team. Observing in a new range of gravitational-wave frequencies is a similar idea, she says. "We won't know what's out there till we look!"

In the team's detector design, nanoparticles are levitated within optical cavities at antinodes (or "bright spots") of standing waves created by lasers. When a gravitational wave passes through, it would change the lengths of the cavities. This change shifts the locations of the laser beam's antinodes and pushes the levitated particles to new locations. While LIGO detects gravitational waves by measuring the changing lengths of its cavities, this new detector would instead measure the motions of the levitated nanoparticles. The experiment requires the ability to sense tiny forces $\left(10^{-21}\right.$ newtons), which Geraci and colleagues have shown is possible in past work.

The advantage of the levitated particle method is that it would be sensitive to higher frequencies of gravitational waves than LIGO is. Because of the way LIGO makes its measurements, its sensitivity is limited by the number of photons in its cavities and gets worse at higher frequencies. But for the levitated particle 
setup, measurement sensitivity is limited by thermal noise of the particles' motions and gets better at higher frequencies.

LIGO looks for signals that are up to about $10 \mathrm{kHz}$ in frequency. The prototype levitated particle system, which will be about one meter in size, could detect signals with frequencies as high as $300 \mathrm{kHz}$ and as low as $10 \mathrm{kHz}$, though with poorer sensitivity than LIGO. If experiments with the 1-m prototype go well, the team hopes to build a 10-m version that Geraci says would be competitive with LIGO's sensitivity even at the $10-\mathrm{kHz}$ range.

With a detector that's sensitive at these high frequencies, the team could look for gravitational waves that would be emitted if axions-candidate dark matter particles that are their own antiparticles-annihilated with each other. If axions exist, researchers think that they would form clouds around black holes in huge numbers, potentially producing enough gravitational waves to be detectable from Earth. Each pair of axions that annihilates in a cloud would produce a gravitational wave with an energy equivalent to the mass of two axions. Though researchers don't know what mass axions would have, they think all axions would have the same mass, meaning gravitational waves with two axions' worth of energy would all have the same frequency.

With a high-frequency gravitational-wave detector, Geraci says, the team would scan over the range of frequencies it can observe, looking for a spike of long-lived gravitational waves at a single frequency from these annihilations. "That would be the kind of smoking gun signature you'd hope to find if that process was really there and the axions really existed," Geraci says. But he explains that the prototype is still in the design and testing phases, and it will probably be a couple of years before it can start taking observations.

Marianna Safronova, a physicist at the University of Delaware who chaired the DAMOP meeting session, says she finds the proposed device very interesting because of its modest size compared to LIGO. But she expects that there will be many challenges, since the device is a completely new type of gravitational-wave detector. "That's why it's important to build the prototype," she says.

This levitated particle system is just one example of a high-precision technique from atomic and optical physics that researchers are adapting to look for new physics beyond the standard model. Other experiments use atomic clocks, atomic magnetometers, and even GPS to look for new particles, new forces, changes in fundamental constants, and more. Those efforts are flourishing, says Safronova, who specializes in high-precision techniques, because these techniques continue to improve. "We really don't know what the limit is."

Erika K. Carlson is a Corresponding Editor for Physics based in Brooklyn, New York. 